\title{
Psychological pressure and compounded errors during elite-level tennis
}

\author{
David J. Harris \\ University of Exeter
}

Michael W. Eysenck,

Royal Holloway University of London

\author{
Samuel J. Vine,
}

University of Exeter

Mark R. Wilson

University of Exeter

Objectives: In the context of Grand Slam tennis, we sought to examine how situational pressure and prior errors can disrupt subsequent performance in elite performers.

Methods: A retrospective analysis of more than 650,000 points across 12 Grand Slam tennis tournaments from 2016-2019 was conducted to identify pressurised in-game moments and unforced errors. A scoring system was used to index situational pressure based on the current match situation (e.g., break points, stage of the match) on a pointby-point basis. The occurrence of performance errors was identified based on double faults and unforced errors, as instances of controllable mistakes. Results: A mixed effects logistic regression model revealed that an increase in the pressure index (a 1-5 score) significantly increased the probability of a performance error $\left(p_{\mathbf{s}}<.001\right)$, as did an error on the preceding point ( $\mathrm{OR}=1.2,95 \% \mathrm{CI}[1.17,1.23], p<.001)$. A multiplicative effect of pressure and prior errors also emerged, as the negative impact of prior errors on performance was greater when situational pressure was already high, in line with the predictions of Attentional Control Theory: Sport (ACTS). Analyses of the distribution of winners and unforced errors across individual players revealed that winning players were as susceptible to pressure and prior errors as losing players. Conclusions: These findings extend our understanding of how ongoing feedback from prior mistakes may further exacerbate the effects of pressure on performance.

Keywords; anxiety; dependency; choking; failure; clutch;

The competitive sporting environment generates psychological pressure, described as 'any factor or combination of factors that increases the importance of performing well' (Baumeister, 1984, pp. 610). These factors include performance-contingent rewards, competition, ego relevance, and audience observation (Baumeister \& Showers, 1986). Research in sport psychology has documented 
how psychological pressure can create the negative emotional state of anxiety (comprised of cognitive worry and physiological arousal; Eysenck, 2013), which subsequently impairs the execution of welllearned skills (Roberts et al., 2019). This impairment has been termed choking and occurs when a performer exhibits a negative response to perceived pressure, despite striving to perform well (Baumeister, 1984; Beilock \& Gray, 2007; Hill et al., 2010). Irrespective of whether this breakdown occurs due to the disruption of automated motor processes or distraction via worry (see, (Payne et al., 2018; Roberts et al., 2017)for recent reviews), there remains a persistent puzzle surrounding who copes and who chokes under pressure (Hill et al., 2010; Otten, 2009).

Attentional Control Theory: Sport (ACTS; Eysenck \& Wilson, 2016), aims to address this gap by not only describing the effect of anxiety on performance, but by considering how anxiety arises in the first place. ACTS maintains the predictions of Attentional Control Theory (ACT; Eysenck et al., 2007) which proposes that anxiety causes increased attention to threat-related cues as a result of a disrupted balance between top-down and bottom-up attentional systems (Cocks et al., 2016; Corbetta \& Shulman, 2002; Wilson, 2008). However, ACTS extends this core mechanism to suggest that the origins of competitive anxiety are rooted in ongoing appraisals of the costs of failure ('what's at stake?') and the probability of failure ('how am I doing?') (Berenbaum, 2010; Martens et al., 1990; see Figure 1). While external factors (e.g., social comparison, monetary reward) might create situational pressure, it is only the appraisal of whether success is important and whether failure is likely, that will lead to the initiation of the experience of anxiety. If the performer sees the cost of failure as low, or unlikely to occur, they may well avoid the anxiety-inducing effect of psychological pressure.

In addition to describing the appraisals that precipitate anxiety, ACTS identifies the important role that momentary errors may play in this process, an issue that has received limited attention within sport psychology. Within the ACTS framework (see Figure 1), the perceived probability of future failure increases as a function of the number of recent failure experiences; primarily mental and physical errors (e.g., Nicholls et al., 2005). Crucially, ACTS predicts an interactive effect, whereby errors are more likely to be attended to and interpreted more negatively when anxiety is already high (i.e., increased attention to threat; Eysenck et al., 2007). Support for this postulate comes from findings in both mainstream cognitive psychology and cognitive neuroscience. For instance, a series of four experiments by Liu, Shen, and $\mathrm{Li}$ (2019) using a dot-probe task demonstrated a positive feedback loop between state anxiety and attentional bias, in which state anxiety directly increased attentional bias towards negative words and an experimentally-induced negative attentional bias increased state anxiety under stressful conditions. This effect of attentional bias on state anxiety (but not the reverse effect) was found to be moderated by cognitive appraisals (see also Basanovic et al., 2020). Additionally, Aarts and Pourtois (2012) found that an electroencephalogram (EEG) eventrelated potential sensitive to the valence of feedback indicated that error monitoring was more 
disrupted in highly anxious individuals. Consequently, the co-occurrence of high levels of psychological pressure and prior performance errors may be a recipe for choking, if individuals are more attuned to failure cues (errors) and appraise them as likely to influence subsequent performance.

A recent study supported this error dependency effect in elite sport (American Football), finding that when one error was made, the probability of making another on the subsequent play increased (Harris et al., 2019). Harris et al. examined all plays from the National Football League (NFL) over seven seasons, using a scoring system to identify the occurrence of high-pressure plays and substantial errors. As has been found previously in laboratory-based (Cooke et al., 2010) and realworld (Hickman \& Metz, 2015; Pocock et al., 2018; Toma, 2017) studies, an increase in performance pressure resulted in more frequent errors. Crucially for the predictions of ACTS, the detrimental effects of psychological pressure and a preceding error also had an interactive effect, causing the greatest disruption when a play was at a crucial moment of the game and preceded by an error. Indeed, the probability that one error would follow another almost doubled from $27 \%$ on low pressure plays to $50 \%$ on high pressure plays (Harris et al., 2019).

The findings of Harris et al. (2019) did not, however, permit examination of individual differences and trait level factors in coping strategies and choking responses that might moderate the effects of situational pressure. One potentially important trait discussed in the sport psychology literature is that of habitual 'clutch' performers (Hibbs, 2010; Otten, 2009; Schweickle et al., 2021). Clutch performers are individuals who customarily respond well to psychological pressure by avoiding choking and even improving their performances (Schweickle et al., 2021; Swann et al., 2017). However, real-world game statistics have not convincingly supported the idea that particular individuals consistently raise their performances under pressure (Birnbaum, 2008). For instance, Solomonov et al. (2015) showed that NBA (National Basketball Association) players commonly thought to be 'clutch players' did not actually improve their shooting percentage in the last 5 minutes of games. However, they did take more shots which may account for the perception of 'clutch'. As such, the existence of habitual positive responders to pressure remains uncertain. 


\section{Figure 1.}

Schematic representation of the bi-directional pressure-performance relationship, as outlined in Attentional Control Theory (Eysenck et al., 2007; dark grey; bottom right) and Attentional Control Theory: Sport (Eysenck \& Wilson, 2016; light grey; top left). Dashed lines indicate a weaker effect. The top left of the model describes how psychological pressure does not lead directly to anxiety but is mediated by cognitive appraisals of cost and probability of failure. The top right of the model depicts a feedback loop from prior performance, illustrating the effect that errors may have on cognitive appraisals.

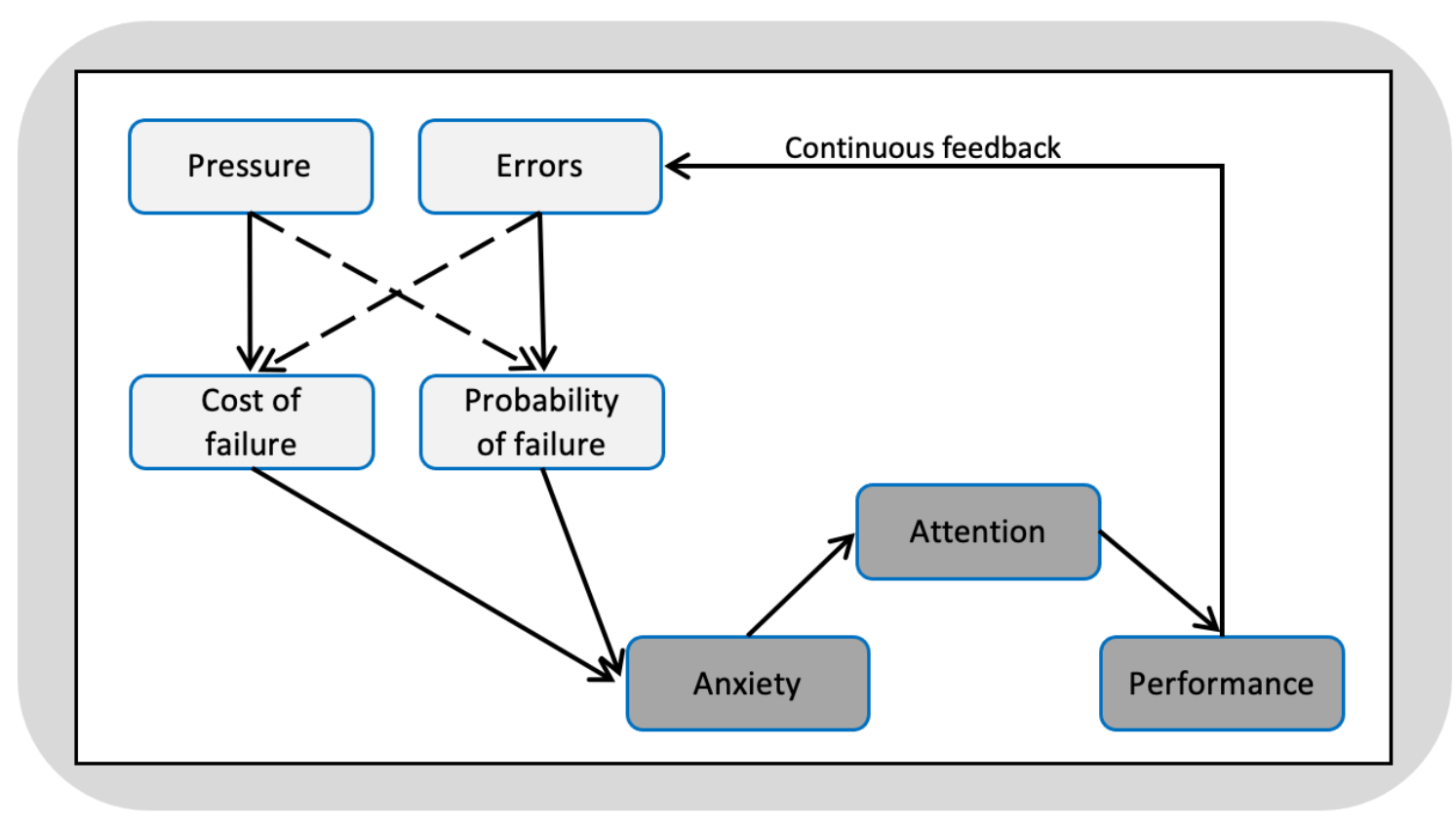

\section{The Present Study}

Examining large, real-world data sets (e.g., as in Harris et al., 2019; Hickman \& Metz, 2015; Toma, 2017) offers greater statistical power and opportunities to test predictions about pressure outside of the artificial laboratory environment, where 'blocked' experimental conditions are unlikely to reveal anything about the drivers of anxiety and its effect on the critical occasions when performance truly matters. For instance, Toma (2017) found evidence of widespread choking in both men's and women's college and professional basketball, with players less likely to successfully execute free throws in the final minutes of close games. Similarly, Hickman and Metz (2015) found that for individual putts taken on the PGA tour, as the amount of money riding on the shot increased (i.e., potential change in earnings), so did the likelihood of a miss. The adoption of existing real-world data sets in the current study allowed us to test the overarching predictions of ACTS (Eysenck \& Wilson, 2016) in relation to pressure, errors, and performance outcomes in the real-world. It is recognised, however, that it is not possible to assess the intervening cognitive mechanisms with this approach (cf. Aarts \& Pourtois, 2012; Liu et al., 2019). Therefore, we do not offer a full test of ACTS, but rather use the performance-related predictions as a basis for examining the effects of pressure and prior errors. 
The aim of the study was to replicate and extend the findings of Harris et al. (2019) in relation to the play-by-play effects of pressure and prior errors on performance in American Football to a point-by-point perspective in Grand Slam tennis tournaments. Grand Slam tournaments were chosen as they represent the most prestigious and lucrative tournaments for elite players, and hence are likely to elicit maximal psychological pressure. The results of Harris et al. were complicated by the nature of team sports (e.g., in how pressure is differentially experienced, and errors attributed). Consequently, we aimed to replicate these findings in an individual sporting endeavour (singles tennis) where unforced errors (rather than good plays by an opponent) could be more easily determined. Based on the predictions of ACTS, and prior findings in American Football (Harris et al., 2019), it was hypothesised that pressure would have a negative effect on performance (i.e., more unforced errors) $\left(\mathrm{H}_{1}\right)$; prior errors would have a negative effect on performance $\left(\mathrm{H}_{2}\right)$; and there would be an interactive effect, such that the effect of a preceding error would be greatest under higher pressure $\left(\mathrm{H}_{3}\right)$. It was also predicted that changes in the rate of unforced errors would be a result of impaired skill execution (choking) rather than a general change in playing strategy (i.e., the balance of risk/reward in shot selection). Therefore, it was expected that changes in unforced errors as a result of pressure or prior errors would not be accompanied by a similar increase in 'winners' (as a measure of higher-risk shots) $\left(\mathrm{H}_{4}\right)$.

To extend previous work using real-world data sets, we also aimed to examine differential responses to psychological pressure and errors to determine whether players who were subsequently successful coped better with pressure and/or responded better to in-game mistakes. Based on realworld data it has previously been suggested that highly-ranked professional tennis players perform particularly well when the stakes are high, as more highly-ranked players beat lower ranked players more frequently when playing in Grand Slam tournaments (Jetter \& Walker, 2015). However, the sum of the evidence for 'clutch' performers is weak (Birnbaum, 2008; Solomonov et al., 2015). Therefore, we tentatively hypothesised that all players would be affected by pressure and prior errors, and that any advantage for subsequently successful players at higher levels of pressure and after errors would be commensurate with their general performance advantage $\left(\mathrm{H}_{5}\right)$.

\section{Methods}

We performed a retrospective analysis of existing data from the four tennis Grand Slam tournaments (Australian Open, French Open, Wimbledon, and US Open). Point-by-point data from grand slam matches (all men's and women's singles matches) between 2016-2019 were scraped from the tournament websites, corresponding to 12 tournaments, 3,552 matches and 658,068 individual points of tennis ${ }^{1}$. Four tournaments - the French Open and Australian Open for 2018 and 2019 - had some missing data (the occurrence of double faults and unforced errors was not recorded) so these

\footnotetext{
${ }^{1}$ https://github.com/JeffSackmann/tennis_slam_pointbypoint
} 
tournaments were excluded from analysis. The relevant variables extracted from the dataset were: the ongoing score in the match (sets and games); which player was serving; which player won the point; and when break points, double faults, unforced errors and clean winners occurred. The full data set is available online and the data included in the analysis, as well as the analysis code, is available from the Open Science Framework (https://osf.io/b4vpd/).

\section{Measures}

\section{Errors}

Errors were operationalised as a double fault or an unforced error, as instances of mistakes that were controllable, and not the direct result of a good shot from the opponent. Both unforced errors and double faults were already coded in the data from the Grand Slam websites and were combined into an overall 'unforced errors' performance measure. Next, points on which the player had made an unforced error on the immediately preceding point were coded as 'post-error' points (i.e., on the previous point within the same game).

\section{Winners}

In tennis, winners are shots that are unreturned by the opposing player and which they do not get their racquet on. Consequently, a winner is not only an instance of successful shot execution, but potentially also a higher risk shot than one hit to where the opponent can reach the ball.

\section{Pressure}

Scoring of psychological pressure was based on a system devised by the authors but derived from similar work (Deutscher et al., 2018; Harris et al., 2019; Hickman \& Metz, 2015; Toma, 2017). From a theoretical perspective, the occurrence of pressure is a result of conditions that increase the importance of performing well (Baumeister, 1984), such as playing in the final of a Grand Slam tournament. Therefore, points that were played towards the end of games, towards the end of sets, and towards the end of matches were deemed to be higher pressure as they had a more direct effect on the outcome of the contest. Additionally, game points and break points (where winning the point would result in winning the game for one of the players) were deemed to add additional pressure, because the importance of performing well was inherently higher than earlier points in a game. Consequently, pressure was assigned in a cumulative manner whereby all points started with a base score of 1 , which was increased when any of the following occurred:

1) It was late in the game (i.e., both players on $30 / 40 / \mathrm{adv}$ );

2) If the opponent could win the set in the game (so 5-4 or 5-1 or 6-5 etc.);

3) The match had gone to a deciding set ( $5^{\text {th }}$ for men, $3^{\text {rd }}$ for women);

4) If opponent had game point;

5) If facing break point. 
Therefore, if a player was 40-30 down on their own serve, in a deciding set, and down 5 games to 4, this would create the maximum pressure score. During data analysis it was observed that the maximum score occurred very infrequently (only when facing a break point coinciding with match point), so the last two pressure categories (scores of 5 or 6 ) were combined to provide a pressure index ranging from 1 to 5, with 5 indicating highest pressure (see Harris et al., 2019).

\section{Data Analysis}

Linear mixed effects models (LMMs) were used to examine the effect of pressure and prior errors on performance, using the lme 4 package for R (Bates et al., 2014). The use of a mixed effects model reflects the assumption that there is likely a range of true effects across participants, matches, and tournaments, from which we aim to estimate the mean. Initially, near maximal models were fitted, using random factors for participant (slope and intercept) and participant nested within tournament (slopes and intercepts) (Barr et al., 2013). The models were then simplified to provide the most parsimonious fit based on Principal Components Analysis, using the RePsychLing package, as described by Bates et al. (2018), and the Akaike information criterion for comparing competing models. We report an odds ratio (OR) as an effect size for the dichotomous outcome variables unforced errors and winners. All analysis scripts and raw data are available from the Open Science Framework (https://osf.io/b4vpd/).

\section{Results}

\section{Errors}

A linear mixed effects model (with player as a random factor) was run to examine the effect of game pressure and prior errors on performance. Firstly, a prior unforced error was found to increase the chance of a further unforced error ( $\mathrm{OR}=1.2,95 \% \mathrm{CI}[1.17,1.23], p<.001)$. Secondly, an increase in pressure to a score of 3,4 , or 5 was also found to increase the rate of unforced errors $(p s<.001)$. Pairwise comparisons, with a Bonferroni-Holm adjustment, indicated the rate of errors at each level of pressure to be significantly different from all others at $p<.001$, apart from levels of 4 and 5 which were only marginally different $(p=.05)$, and levels of 2 and 1 which were not significantly different $(p=.80)$ (see Figure 3 Panel A).

Significant interaction effects at pressure levels of $2(p=.03), 3(p=.008)$ and $4(p<.001)$ were also found, which indicated that the effect of a prior error was exacerbated as pressure increased. Pairwise tests with Bonferroni-Holm correction indicated that the likelihood of an error was significantly higher on post-error points for all levels of pressure score $(p \mathbf{s}<.001)$ (see Figure 2 Panel A and Figure 3 Panel B). 


\section{Table 1}

Odds ratios (and their 95\% CIs) for the fixed effects split by level

\begin{tabular}{lllll}
\hline & OR & $\begin{array}{c}\text { CI low } \\
(\mathbf{2 . 5 \% )}\end{array}$ & $\begin{array}{l}\text { CI high } \\
(\mathbf{9 7 . 5 \% )}\end{array}$ & p-value \\
\hline Intercept & 0.18 & 0.18 & 0.19 & $<.0001^{* * *}$ \\
Pressure 2 & 1.01 & 0.99 & 1.02 & .299 \\
Pressure 3 & 1.14 & 1.12 & 1.16 & $<.0001^{* * *}$ \\
Pressure 4 & 1.47 & 1.41 & 1.54 & $<.0001^{* * *}$ \\
Pressure 5 & 1.86 & 1.59 & 2.18 & $<.0001^{* * *}$ \\
Post error & 1.20 & 1.18 & 1.23 & $<.0001^{* * *}$ \\
Pressure 2 * Post error & 1.04 & 1.01 & 1.08 & $.025^{*}$ \\
Pressure 3 * Post error & 1.07 & 1.02 & 1.12 & $.008^{* *}$ \\
Pressure 4 * Post error & 1.24 & 1.13 & 1.36 & $<.001^{* * *}$ \\
Pressure 5 * Post error & 1.33 & 0.93 & 1.90 & .12 \\
\hline
\end{tabular}

Note: reference categories for the odds ratios are a pressure score of 1 and no error. ${ }^{*} \mathrm{p}<.05,{ }^{*} \mathrm{p}<.01$, $* * * \mathrm{p}<.001$

\section{Figure 2}

Plotted point estimate (and 95\% CIs) for effect of model predictors on unforced errors (Panel A) and winners (Panel B).
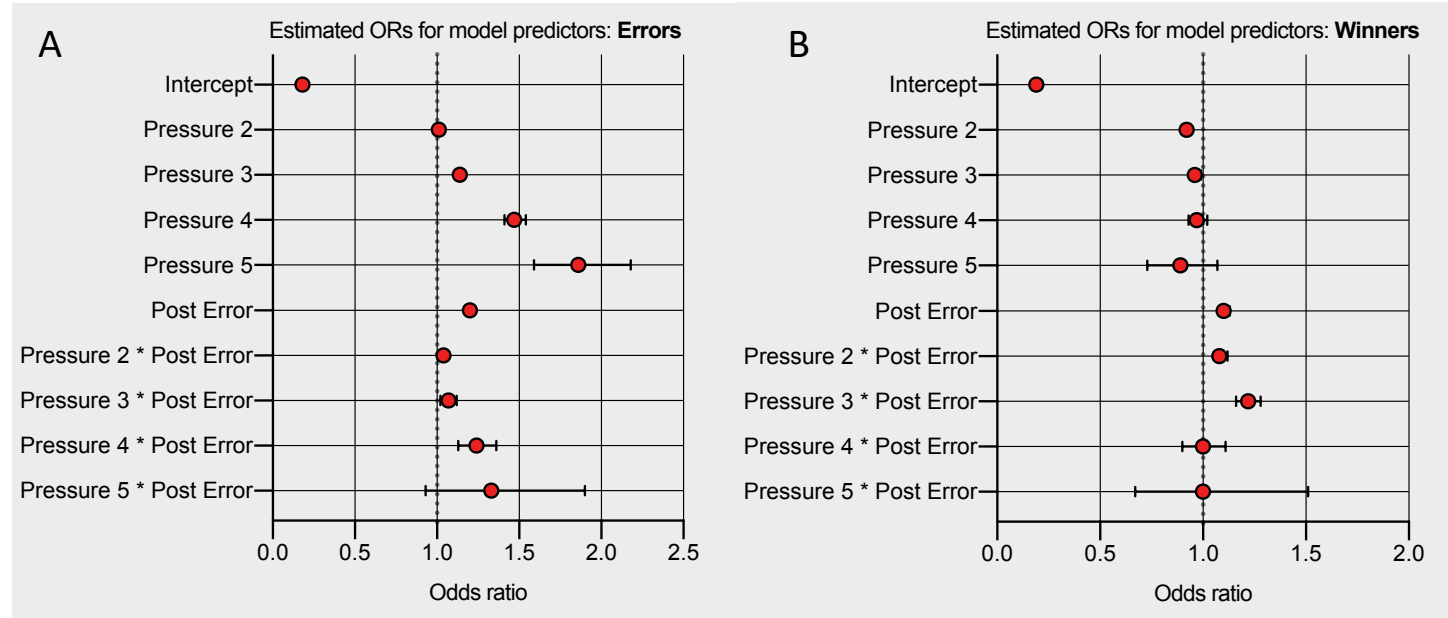

Note: Reference categories for the odds ratios are a pressure score of 1 and no error

\section{Winners}

To examine whether changes in the rate of unforced errors across levels of pressure was confounded by a change in playing strategy (i.e., were players just less conservative in their play following an error when under pressure?) we also examined the rate at which players hit winners (i.e., unreturnable shots). A linear mixed effects model (with player as a random factor) indicated that winners were less frequent at pressure scores of 2 (OR=0.92, 95\% CI [0.91, 0.94], $p<.001)$ or 3 $(\mathrm{OR}=0.96,95 \% \mathrm{CI}[0.94,0.99], p=.001)$ compared to the reference category (index of 1$)$, but there were no significant effects at other levels $(p s>.22)$ and no consistent pattern of effect (see Figure 2 
Panel B and Figure 3 Panel B). This result confirms that changes in error rates under pressure could not be explained by a change to a more high-risk strategy. Winners were, however, found to be more frequent on points following an error ( $\mathrm{OR}=1.10,95 \% \mathrm{CI}[1.08,1.13], p<.001)$ (see Figure 2 Panel B). Small interaction effects were found at pressure levels of 2 ( $\mathrm{OR}=1.08,95 \% \mathrm{CI}[1.05,1.12], p<.001)$ and $3(\mathrm{OR}=1.22,95 \% \mathrm{CI}[1.16,1.28], p<.001)$, but not at 4 or $5(p \mathrm{~s}>.97)$. Pairwise tests with Bonferroni-Holm correction indicated that the likelihood of a winner was significantly higher on posterror points at low levels of pressure (1,2, and $3 ; p s<.005)$, but not higher levels (4 and $5 ; p s>.12$ ).

\section{Figure 3}

Probability of unforced errors (mean and 95\% CIs) across pressure score (Panel A) and probability of unforced errors (bars) and winners (points) across pressure score, split by preceding error (Panel B).
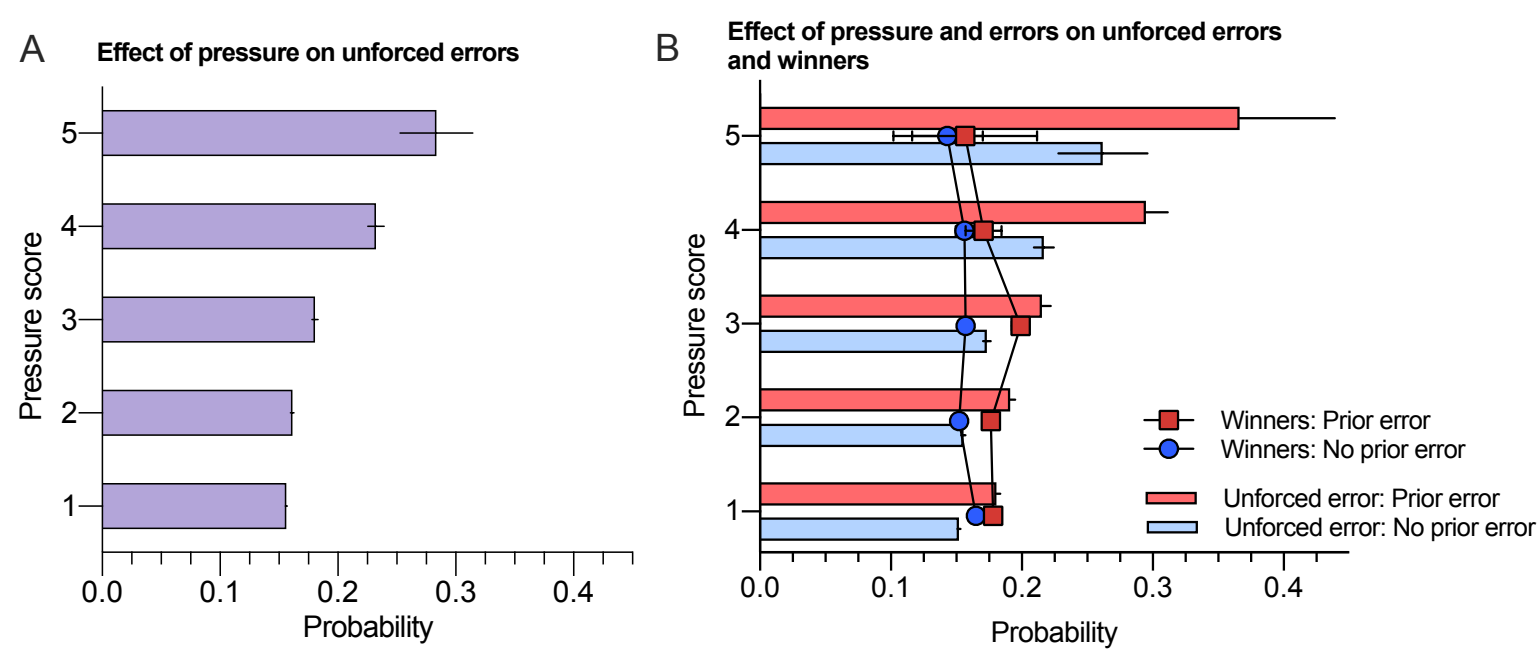

\section{Individual Responses to Pressure and Errors}

To explore potential differential effects in responding to psychological pressure and prior errors, we tested whether there was a clear clustering of datapoints (see Figure 4) representing high rates of winners and low rates of unforced errors for players who subsequently won their match (i.e., successful), compared to those who lost (i.e., unsuccessful). An algorithm called the interpoint distance (IPD) test (see Marozzi et al., 2020) was used to determine whether the underlying distributions of errors/winners among successful and unsuccessful players were statistically different. IPD testing enables comparisons of the mean, variance, and underlying distributions of highdimensional data based on the Euclidean distance between points, free from any assumptions about underlying distributions. Additionally, a 95\% confidence interval (CI) bounding ellipse of the data for successful and unsuccessful players was calculated, and the degree of overlap between the two ellipses was determined using the maximum likelihood method. For all points across all games (Figure 4 Panel A) the IPD test indicated that the underlying distributions of winners to unforced errors for successful versus unsuccessful players were significantly different $(p<.001)$, with $54.8 \%$ 
overlap in the 95\% CI ellipses. For higher-pressure points (index of 3+; Figure 4 Panel B), distributions were again significantly different $(p<.001)$ with a similar level of overlap (55.8\%). For points following an error (Figure 4 Panel C) the IPD test indicated that distributions were again significantly different $(p<.001)$ but showed a greater degree of overlap (67.5\%). Finally, for high pressure points that also followed an error (Figure 4 Panel D), the distributions remained significantly different $(p<.001)$, but showed a further increase in overlap (76.8\%). Consequently, there was no evidence for differential effects of pressure and errors: winning players generally had a better ratio of winners to unforced errors, but this difference did not change for high pressure points and was actually reduced for points after an error, particularly when coinciding with high pressure. 


\section{Figure 4}

Jittered scatter plots (with marginal distribution histogram) of the rate of unforced errors against the rate of winners for all points in all games (A), for the higher pressure $(3+$ score) points (B) for the post error points (C), and for the post error points coinciding with high pressure (D) colour coded by match winner. 95\% confidence interval error ellipses, and the percentage overlap between winners/losers have been calculated.
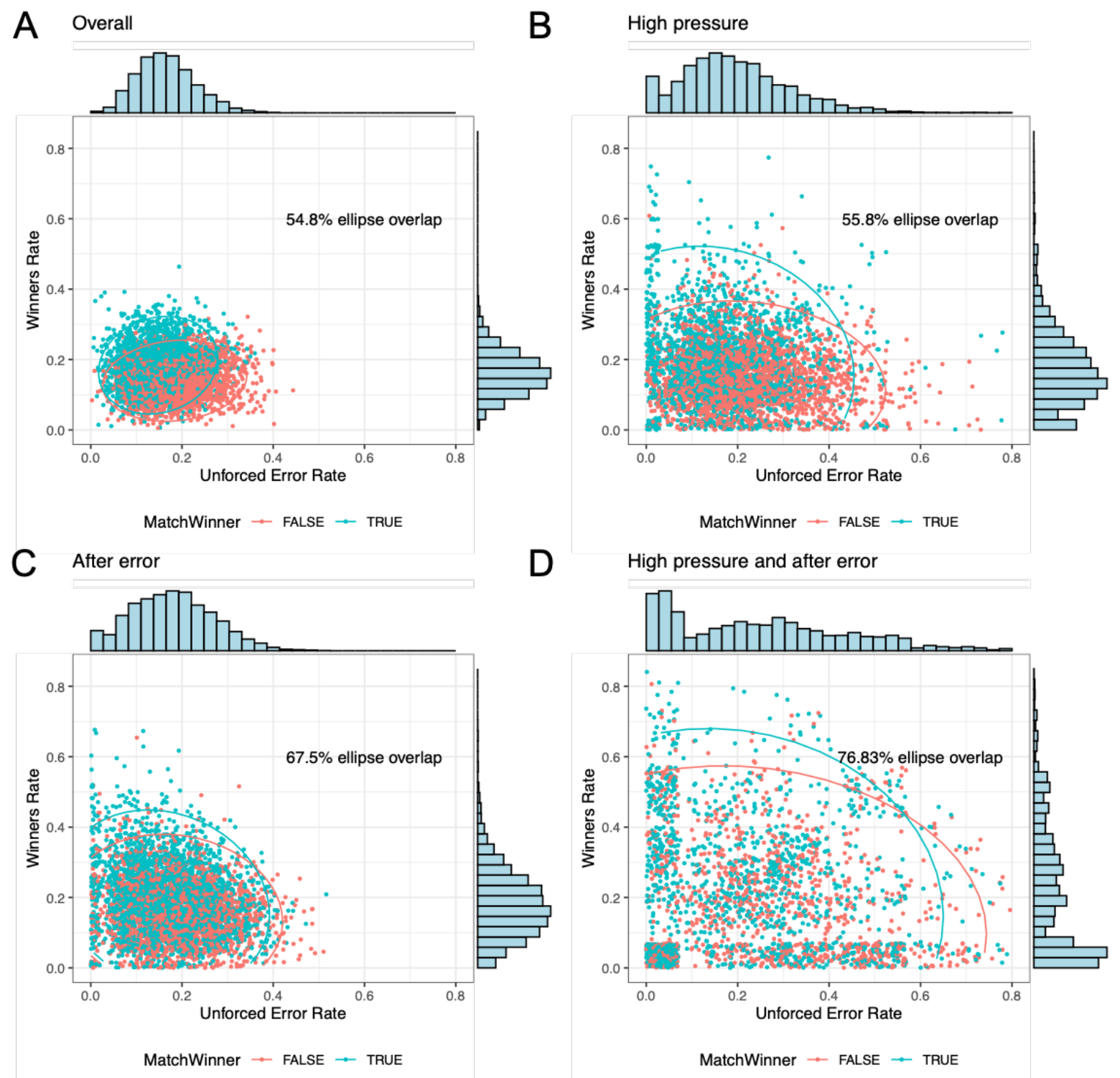

\section{Discussion}

Direct testing of predictions derived from theory is important for refining and developing better theoretical models in psychology (Ferguson \& Heene, 2012). In the context of elite-level tennis, we sought to examine the effects of psychological pressure and prior errors on subsequent performance. In particular, we aimed to test the overarching predictions of ACTS (Eysenck \& Wilson, 2016) that pressure and prior errors would have multiplicative effects on performance. In line with our first hypothesis, there was a clear effect of pressure, with more unforced errors occurring as the 
pressure index increased. There was little difference in error rate between pressure scores of 1 and 2, but all subsequent increases in pressure resulted in a corresponding increase in errors (see Figure 3 Panel A). The unforced error rate for the highest-pressure points $(0.28 \pm 0.45)$ was 1.75 times that of low-pressure points $(0.16 \pm 0.36)$. The observed effect of pressure on performance is in line with prior work examining real-world data sets in basketball (Toma, 2017) and golf (Hickman \& Metz, 2015), although a contrasting finding has been reported by Deutscher et al. (2018) in darts.

Deutscher et al. (2018) suggested that high-level darts players actually perform better under pressure, as evidenced by improved performance when faced with more challenging game situations: a three-dart finish as opposed to a one-dart finish (but with three opportunities) when the opponent can also finish on their next visit. However, Deutscher et al. interpreted pressure solely as a function of absolute probability of successful outcome, ignoring the role that cost of failure plays in the perception of pressure (Berenbaum, 2010). While hitting three specific shots to finish is clearly much harder than only hitting one, the expectation of success is very different. One of the major costs for a sporting performer is the effect that failure has on their own self-concept/self-esteem (referred to as 'ego threat'; Leary et al., 2009), therefore it could be argued that one-dart finishes are likely to be more pressurised than three-dart finishes given the increased expectation of success. Considered in this light, professional darts players may not in fact be excelling under pressure, but choking, as has been shown in basketball (Toma, 2017), golf (Hickman \& Metz, 2015), and American Football (Harris et al., 2019).

Our second hypothesis concerned the effect of preceding errors on subsequent performance and the idea that there may be a degree of dependence between successive points. The results indicated that the rate of unforced errors was significantly increased on points following an error and hence supported the potentially detrimental error feedback effect (Aarts \& Pourtois, 2012; Baumeister et al., 2001; Eysenck \& Wilson, 2016; Harris et al., 2019). Our third hypothesis, predicting an interactive effect between pressure and errors, was also supported. At pressure index scores of 2, 3, and 4 a significant interaction effect, over and above the additive effects of pressure and errors, was observed. This interaction was not detected at a pressure score of $5(p=.12)$, however the subset of data at the highest level of pressure was much sparser than other levels. Figure 3 (Panel B) reflects that the interaction pattern appears to continue to the pressure index of 5 , but with a much less certain point estimate, which may explain the lack of a significant effect.

The observed interactive effect of pressure and errors is in line with the predictions outlined in ACTS where the perceived probability of failure is likely to increase after an error (i.e., dependence) and interacts with the cost of failure (which is greater at critical points in a match) to raise performance anxiety. Previous investigations of the human performance-monitoring system have demonstrated how error detection serves as a signal to adapt and improve ongoing behaviour (Botvinick et al., 2001). Yet, in the context of high anxiety this process may prove maladaptive. 
Masaki et al. (2017) have previously shown how sports anxious individuals exhibit neural markers of abnormal error monitoring when performance was evaluated. While the appraisal of errors could not be measured in the present work, when considered together with previous findings that anxiety increases attention to threat (Bar-Haim et al., 2007), increases error monitoring (Moser et al., 2013), and leads to difficulty disengaging with emotionally negative information (Fox et al., 2001), the cooccurrence of high-pressure and a prior error may set off a chain of events where negative stimuli (e.g., previous failure and the prospect of future failure) are fixated on, causing further anxiety and further mistakes. This sequencing of anxiety, error monitoring, and attention to threat should be further investigated in future research on performance under pressure.

While we were unable to affirm the intervening cognitive mechanisms relating to attentional bias and cognitive appraisals outlined in ACTS, the observed interaction effect cannot simply be explained by a shift towards a more high-risk strategy (i.e., more unforced errors and winners) under pressure. Our analysis of 'winners' did not reveal any effect of pressure nor an interaction effect that would indicate a high-risk strategy in response to pressure. Indeed, the opposite - a more conservative approach to avoid further errors - would seem more plausible and future work may wish to examine potential in-game changes in strategy, particularly in relation to the cognitive appraisal of prior mistakes and their perceived probability and costs (Berenbaum, 2010). Consequently, the effect of pressure on unforced errors is most likely related to a skill breakdown, as described in theories of anxiety and pressure on sporting performance (Beilock \& Carr, 2001; Eysenck \& Wilson, 2016; Masters \& Maxwell, 2008; Nieuwenhuys \& Oudejans, 2012; Vine et al., 2016; Masters, 1992; Nieuwenhuys \& Oudejans, 2012). Future work may wish to examine the cognitive appraisal of errors in more detail by collecting self-reported appraisals after experimentally manipulated error feedback. This would enable a more comprehensive test of the predictions of ACTS (see Figure 1) in terms of how the appraisal of errors relates to changes in attention, and subsequently performance.

To examine whether the observed pressure and error effects were universal, or whether subsequently successful players were better able to avoid additional unforced errors, we examined the distributions of winners and unforced errors using an interpoint distance test $\left(\mathrm{H}_{5}\right)$. Research into 'clutch' performance has suggested that some individuals cope better under psychological pressure (Otten, 2009), but our findings provided no support for this effect. Predictably, successful players were found to have a much better ratio of winners to unforced errors across all points (Figure 4 Panel A). However, for higher pressure points the distributions of winners to unforced errors for successful/unsuccessful players (Figure 4 Panel B) was very similar to the overall distributions, indicating that successful players simply maintained their general advantage under pressure and did not somehow 'raise their game'. On post-error points (Figure 4 Panel C), the distinction between successful and unsuccessful players was much reduced, suggesting that all players struggled to recover from errors. Further, on post-error points that were also higher pressure, the distinction 
between successful and unsuccessful players was smaller still, again suggesting that the co-occurrence of pressure and a prior error had a damaging effect, regardless of the player's ability. Clearly, individualised responses to sporting situations will determine whether performers perceive any pressure and how they will respond in performance terms (Schweickle et al., 2021), but as our data was anonymised, we could not explore potentially interesting individual factors (e.g., player ranking or recent performance history). However, these findings provided no evidence for any widespread 'clutch' effect in winning players, and instead supported the ubiquity of the pressure and error feedback effects.

It is important to note that our findings contrast with previous work which has reported higher-ranked professional tennis players to improve their winning percentage in clutch situations (Jetter \& Walker, 2015). However, the strongest evidence for clutch effects reported by Jetter and Walker was that higher-ranked players increased their probability of beating lower ranked players when playing in Grand Slam tournaments. An alternative interpretation of Jetter and Walker's data is, however, that highly ranked players simply under-performed in less important tournaments, where their motivation may have been lower. Further, competing in best-of- 3 set matches, as opposed to 5 (for men) in non-grand slam tournaments increases the odds of extreme results. Jetter and Walker do report that the evidence for more acute within-game instances of clutch performance - that higher ranked players would excel in tie breaks and deciding sets - was weak in comparison to the broader effect of playing in Grand Slam events. Additionally, Jetter and Walker were able to identify the top ranked players in the world which was not possible in the current data set. The clutch abilities of these super-elite players may be different to the broader successful / unsuccessful effects which we report here.

Nonetheless, our findings do question the idea that subsequently successful players (on average better players) responded more positively to pressure, and instead demonstrate that the effects of situational pressure and prior mistakes affect even the most elite athletes. Indeed, the weight of evidence is strongly in favour of an overall performance degradation under pressure (Harris et al., 2019; Hickman \& Metz, 2015; Toma, 2017), and the notion of 'clutch players' may be a cognitive bias present in observers rather than a real effect (Solomonov et al., 2015). Individuals commonly thought to be 'clutch' players - Michael Jordan, Tom Brady, Wayne Gretsky - might well perform at important moments simply because of their general superiority, not because they are immune to the effects of pressure.

One of the practical implications of the present findings is a greater appreciation of the detrimental impact prior errors can have and the need for athletes to develop strategies to mitigate against error dependency effects. While it might be worthwhile to allow performers to commit (and then correct) errors as they are learning skills (Metcalfe, 2017), the fact that anxious apprehension is associated with exaggerated error monitoring (Moser et al., 2013) means that the perception of errors 
can change in high stakes environments. As outlined in ACTS, the cognitive appraisal of an error determines the subsequent effect on anxiety and performance, via its influence on the perceptions of the probability of failure (Berenbaum, 2010). Therefore, developing the ability to respond more positively to errors may be crucial in breaking the feedback loop between attentional bias to threat cues, state anxiety, and cognitive appraisals of threat (Eysenck \& Wilson, 2016; Liu et al., 2019). Instances of athletes choking under pressure are often linked to an initial error that induces anxiety, overthinking, and a detrimental focus of attention on skill execution (Hill et al., 2010; Williams \& Wigmore, 2020). While the present dataset indicated that even winning players struggled in the wake of an error, the ability to simply forget these errors might be a highly valuable mental skill.

This type of post-error recovery is even quantified for the PGA tour as a 'bounce back' percentage ${ }^{2}$; reflecting the frequency by which a player follows up an over par score on a hole with an under-par score on the subsequent hole. By way of a real-world example, Annika Sörenstam (the $3^{\text {rd }}$ most successful golfer of all time on the Ladies PGA tour) reports that she barely remembers making an error; after a bad shot she would conduct a brief analysis of the mistake, then move on and focus on the 'now shot' (Williams \& Wigmore, 2020). Incidentally, the greater length of time between shots in golf compared to tennis may make forgetting easier, and therefore sport type may be an important moderator of this relationship. Frameworks like ACTS can potentially provide a basis for developing practical techniques to avoid negative appraisals of errors, which can otherwise influence fragile performance states like sport confidence (Beaumont et al., 2015). For instance, techniques such as pre-shot routines and constructive self-talk may help to develop this form of 'error amnesia' (Harris et al., 2019), and robust sport confidence (Thomas et al., 2011) as described by Sörenstam.

The benefits of pre-shot routines for performing under pressure are varied and not fully understood (e.g., see Cotterill, 2010; Mesagno \& Mullane-Grant, 2010). However, recent research in cognitive neuroscience suggests that even arbitrary rituals can dull the neural response to performance failure and buffer against uncertainty and anxiety (Hobson et al., 2017). In the language of ACTS, this means that as the cost of failure increases (as pressure is raised) pre-shot routines may disrupt the error feedback cycle that strongly influences perceptions of failure probability. However, as routines are more easily applicable to self-paced skills (e.g., the tennis serve in our data), self-talk interventions, focusing on directing attentional control 'in the moment', may be more appropriate for skills when time constraints are more pronounced (e.g., rally strokes and volleys in our data). Again, the self-regulatory benefits of intentionally using self-talk are numerous; including directing attentional focus, enhancing confidence, regulating effort, and controlling emotional and cognitive reactions (Latinjak et al., 2019; Theodorakis et al., 2008). In the specific context of reducing attention towards potentially threatening failure signals, there is some evidence that self-talk interventions

\footnotetext{
${ }^{2}$ https://www.pgatour.com/stats/stat.160.html
} 
might be useful. Schüler and Langens (2007) tested the use of self-talk strategies as a means for buffering against the negative effects of psychological crisis ('hitting the wall') in non-professional runners completing a marathon. They reported that among runners who experienced hitting the wall, those using self-talk coped better than those in a control group. Consequently, self-talk may be an effective intervention for both regulating responses to pressure and mitigating against the negative feedback cycle of performance errors (Cooper et al., 2020).

\section{Conclusions}

In summary, the present study aimed to examine the negative effects of situational pressure and prior errors on subsequent performance in elite tennis. The results replicate those reported by Harris et al. (2019) in American Football; that both situational pressure and prior errors interact to induce further performance breakdowns. The present findings extend this work to a new sport that avoids the complication of team performance. The findings further clarify the likely momentary conditions where performance might be disrupted, in even the most skilled of athletes, although it is possible the few top players in the world do not show these effects. In particular, the results speak to the power of pressure and prior errors, and the feedback loop that they may form with ongoing cognitive appraisals of the probability and costs of success and failure. However, while the broader performance feedback effects postulated in ACTS are supported, we cannot speak to the intervening mechanisms here. Therefore, future work should attempt the difficult task of examining how momentto-moment fluctuations in meaningful pressure affects cognitive appraisals of the probability of success and failure, and how these appraisals relate to changes in anxiety, attention, and performance. 


\section{References}

Aarts, K., \& Pourtois, G. (2012). Anxiety disrupts the evaluative component of performance monitoring: An ERP study. Neuropsychologia, 50(7), 1286-1296.

https://doi.org/10.1016/j.neuropsychologia.2012.02.012

Bar-Haim, Y., Lamy, D., Pergamin, L., Bakermans-Kranenburg, M. J., \& van IJzendoorn, M. H. (2007). Threat-related attentional bias in anxious and nonanxious individuals: A meta-analytic study. Psychological Bulletin, 133(1), 1-24. https://doi.org/10.1037/0033-2909.133.1.1

Barr, D. J., Levy, R., Scheepers, C., \& Tily, H. J. (2013). Random effects structure for confirmatory hypothesis testing: Keep it maximal. Journal of Memory and Language, 68(3), 255-278. https://doi.org/10.1016/j.jml.2012.11.001

Basanovic, J., Kaiko, I., \& MacLeod, C. (2020). Change in Attentional Control Predicts Change in Attentional Bias to Negative Information in Response to Elevated State Anxiety. Cognitive Therapy and Research. https://doi.org/10.1007/s10608-020-10176-3

Bates, D., Kliegl, R., Vasishth, S., \& Baayen, H. (2018). Parsimonious Mixed Models. ArXiv:1506.04967 [Stat]. http://arxiv.org/abs/1506.04967

Bates, D., Mächler, M., Bolker, B., \& Walker, S. (2014). Fitting linear mixed-effects models using lme4. ArXiv:1406.5823 [Stat]. http://arxiv.org/abs/1406.5823

Baumeister, R. F. (1984). Choking under pressure: Self-consciousness and paradoxical effects of incentives on skillful performance. Journal of Personality and Social Psychology, 46(3), 610620.

Baumeister, R. F., Bratslavsky, E., Finkenauer, C., \& Vohs, K. D. (2001). Bad Is Stronger Than Good. Review of General Psychology, 5(4), 323-370. https://doi.org/10.1037/1089-2680.5.4.323

Baumeister, R. F., \& Showers, C. J. (1986). A review of paradoxical performance effects: Choking under pressure in sports and mental tests. European Journal of Social Psychology, 16(4), 361383. https://doi.org/10.1002/ejsp.2420160405

Beaumont, C., Maynard, I. W., \& Butt, J. (2015). Effective Ways to Develop and Maintain Robust Sport-Confidence: Strategies Advocated by Sport Psychology Consultants. Journal of Applied Sport Psychology, 27(3), 301-318. https://doi.org/10.1080/10413200.2014.996302

Beilock, S. L., \& Carr, T. H. (2001). On the fragility of skilled performance: What governs choking under pressure. Journal of Experimental Psychology: General, 130, 701725.

Beilock, S. L., \& Gray, R. (2007). Why do athletes choke under pressure? In Handbook of sport psychology, 3rd ed (pp. 425-444). John Wiley \& Sons, Inc.

Berenbaum, H. (2010). An initiation-termination two-phase model of worrying. Clinical Psychology Review, 30(8), 962-975. https://doi.org/10.1016/j.cpr.2010.06.011

Birnbaum, P. (2008). Clutch hitting and the cramer test. Baseball Research Journal, 37, 71-75.

Botvinick, M. M., Braver, T. S., Barch, D. M., Carter, C. S., \& Cohen, J. D. (2001). Conflict monitoring and cognitive control. Psychological Review, 108(3), 624-652. https://doi.org/10.1037/0033-295X.108.3.624

Cocks, A. J., Jackson, R. C., Bishop, D. T., \& Williams, A. M. (2016). Anxiety, anticipation and contextual information: A test of attentional control theory. Cognition and Emotion, 30(6), 1037-1048. https://doi.org/10.1080/02699931.2015.1044424

Cooke, A., Kavussanu, M., McIntyre, D., \& Ring, C. (2010). Psychological, muscular and kinematic factors mediate performance under pressure. Psychophysiology, 47(6), 1109-1118.

https://doi.org/10.1111/j.1469-8986.2010.01021.x 
Cooper, K. B., Wilson, M. R., \& Jones, M. I. (2020). Fast talkers? Investigating the influence of selftalk on mental toughness and finish times in 800-meter runners. Journal of Applied Sport Psychology, O(0), 1-19. https://doi.org/10.1080/10413200.2020.1735574

Corbetta, M., \& Shulman, G. L. (2002). Control of Goal-Directed and Stimulus-Driven Attention in the Brain. Nature Reviews Neuroscience, 3(3), 201-215.

Cotterill, S. (2010). Pre-performance routines in sport: Current understanding and future directions. International Review of Sport and Exercise Psychology, 3(2), 132-153. https://doi.org/10.1080/1750984X.2010.488269

Deutscher, C., Ötting, M., Langrock, R., Gehrmann, S., Schneemann, S., \& Scholten, H. (2018). Very Highly Skilled Individuals Do Not Choke Under Pressure: Evidence from Professional Darts. ArXiv:1809.07659 [Stat]. http://arxiv.org/abs/1809.07659

Eysenck, M. W., Derakshan, N., Santos, R., \& Calvo, M. G. (2007). Anxiety and cognitive performance: Attentional control theory. Emotion, 7(2), 336-353. https://doi.org/10.1037/15283542.7.2.336

Eysenck, Michael W. (2013). Anxiety: The Cognitive Perspective. Psychology Press.

Eysenck, M.W., \& Wilson, M. R. (2016). Sporting performance, pressure and cognition: Introducing attentional control theory: Sport. In D. Groome \& M. Eysenck (2nd ed). An introduction to applied cognitive psychology. (Pp. 329-350). London: Routledge.

Ferguson, C. J., \& Heene, M. (2012). A Vast Graveyard of Undead Theories: Publication Bias and Psychological Science's Aversion to the Null. Perspectives on Psychological Science, 7(6), 555-561. https://doi.org/10.1177/1745691612459059

Fox, E., Russo, R., Bowles, R., \& Dutton, K. (2001). Do threatening stimuli draw or hold visual attention in subclinical anxiety? Journal of Experimental Psychology: General, 130(4), 681700. https://doi.org/10.1037/0096-3445.130.4.681

Harris, D. J., Vine, S. J., Eysenck, M. W., \& Wilson, M. R. (2019). To err again is human: Exploring a bidirectional relationship between pressure and performance failure feedback. Anxiety, Stress, \& Coping, 32(6), 670-678. https://doi.org/10.1080/10615806.2019.1643459

Hibbs, D. (2010). A Conceptual Analysis of Clutch Performances in Competitive Sports. Journal of the Philosophy of Sport, 37(1), 47-59. https://doi.org/10.1080/00948705.2010.9714765

Hickman, D. C., \& Metz, N. E. (2015). The impact of pressure on performance: Evidence from the PGA TOUR. Journal of Economic Behavior \& Organization, 116, 319-330. https://doi.org/10.1016/j.jebo.2015.04.007

Hill, D. M., Hanton, S., Matthews, N., \& Fleming, S. (2010). Choking in sport: A review. International Review of Sport and Exercise Psychology, 3(1), 24-39. https://doi.org/10.1080/17509840903301199

Hobson, N. M., Bonk, D., \& Inzlicht, M. (2017). Rituals decrease the neural response to performance failure. PeerJ, 5. https://doi.org/10.7717/peerj.3363

Janelle, C. M. (2002). Anxiety, arousal and visual attention: A mechanistic account of performance variability. Journal of Sports Sciences, 20(3), 237-251. https://doi.org/10.1080/026404102317284790

Jetter, M., \& Walker, J. K. (2015). Game, set, and match: Do women and men perform differently in competitive situations? Journal of Economic Behavior \& Organization, 119, 96-108. https://doi.org/10.1016/j.jebo.2015.07.017

Latinjak, A. T., Torregrossa, M., Comoutos, N., Hernando-Gimeno, C., \& Ramis, Y. (2019). Goaldirected self-talk used to self-regulate in male basketball competitions. Journal of Sports Sciences, 37(12), 1429-1433. https://doi.org/10.1080/02640414.2018.1561967 
Leary, M. R., Terry, M. L., Batts Allen, A., \& Tate, E. B. (2009). The Concept of Ego Threat in Social and Personality Psychology: Is Ego Threat a Viable Scientific Construct? Personality and Social Psychology Review, 13(3), 151-164. https://doi.org/10.1177/1088868309342595

Liu, J., Shen, K., \& Li, H. (2019). How state anxiety and attentional bias interact with each other: The moderating effect of cognitive appraisal. Attention, Perception, \& Psychophysics, 81(3), 694706. https://doi.org/10.3758/s13414-018-01650-y

Marozzi, M., Mukherjee, A., \& Kalina, J. (2020). Interpoint distance tests for high-dimensional comparison studies. Journal of Applied Statistics, 47(4), 653-665. https://doi.org/10.1080/02664763.2019.1649374

Martens, R., Vealey, R. S., \& Burton, D. (1990). Competitive Anxiety in Sport. Human Kinetics.

Masaki, H., Maruo, Y., Meyer, A., \& Hajcak, G. (2017). Neural Correlates of Choking Under Pressure: Athletes High in Sports Anxiety Monitor Errors More When Performance Is Being Evaluated. Developmental Neuropsychology, 42(2), 104-112. https://doi.org/10.1080/87565641.2016.1274314

Masters, R., \& Maxwell, J. (2008). The theory of reinvestment. International Review of Sport and Exercise Psychology, 1(2), 160-183. https://doi.org/10.1080/17509840802287218

Masters, R. S. W. (1992). Knowledge, knerves and know-how: The role of explicit versus implicit knowledge in the breakdown of a complex motor skill under pressure. British Journal of Psychology, 83(3), 343-358. https://doi.org/10.1111/j.2044-8295.1992.tb02446.x

Mesagno, C., \& Mullane-Grant, T. (2010). A Comparison of Different Pre-Performance Routines as Possible Choking Interventions. Journal of Applied Sport Psychology, 22(3), 343-360. https://doi.org/10.1080/10413200.2010.491780

Metcalfe, J. (2017). Learning from Errors. Annual Review of Psychology, 68(1), 465-489. https://doi.org/10.1146/annurev-psych-010416-044022

Moser, J., Moran, T., Schroder, H., Donnellan, B., \& Yeung, N. (2013). On the relationship between anxiety and error monitoring: A meta-analysis and conceptual framework. Frontiers in Human Neuroscience, 7 . https://doi.org/10.3389/fnhum.2013.00466

Nicholls, A. R., Holt, N. L., Polman, R. C. J., \& James, D. W. G. (2005). Stress and Coping Among International Adolescent Golfers. Journal of Applied Sport Psychology, 17(4), 333-340. https://doi.org/10.1080/10413200500313644

Nieuwenhuys, A., \& Oudejans, R. R. D. (2012). Anxiety and perceptual-motor performance: Toward an integrated model of concepts, mechanisms, and processes. Psychological Research, 76(6), 747759. https://doi.org/10.1007/s00426-011-0384-x

Otten, M. (2009). Choking vs. Clutch Performance: A Study of Sport Performance under Pressure. Journal of Sport and Exercise Psychology, 31(5), 583-601. https://doi.org/10.1123/jsep.31.5.583

Payne, K. L., Wilson, M. R., \& Vine, S. J. (2018). A systematic review of the anxiety-attention relationship in far-aiming skills. International Review of Sport and Exercise Psychology, O(0), 1-31. https://doi.org/10.1080/1750984X.2018.1499796

Pocock, C., Bezodis, N. E., Davids, K., \& North, J. S. (2018). Hot hands, cold feet? Investigating effects of interacting constraints on place kicking performance at the 2015 Rugby Union World Cup. European Journal of Sport Science, 18(10), 1309-1316. https://doi.org/10.1080/17461391.2018.1486459

Roberts, L. J., Jackson, M. S., \& Grundy, I. H. (2017). Choking under pressure: Illuminating the role of distraction and self-focus. International Review of Sport and Exercise Psychology, 0(0), 1-21. https://doi.org/10.1080/1750984X.2017.1374432 
Schüler, J., \& Langens, T. A. (2007). Psychological Crisis in a Marathon and the Buffering Effects of Self-Verbalizations1. Journal of Applied Social Psychology, 37(10), 2319-2344. https://doi.org/10.1111/j.1559-1816.2007.00260.x

Schweickle, M. J., Vella, S. A., \& Swann, C. (2021). Exploring the "clutch" in clutch performance: A qualitative investigation of the experience of pressure in successful performance. Psychology of Sport and Exercise, 54, 101889. https://doi.org/10.1016/j.psychsport.2021.101889

Solomonov, Y., Avugos, S., \& Bar-Eli, M. (2015). Do clutch players win the game? Testing the validity of the clutch player's reputation in basketball. Psychology of Sport and Exercise, 16, 130-138. https://doi.org/10.1016/j.psychsport.2014.10.004

Swann, C., Crust, L., Jackman, P., Vella, S. A., Allen, M. S., \& Keegan, R. (2017). Performing under pressure: Exploring the psychological state underlying clutch performance in sport. Journal of Sports Sciences, 35(23), 2272-2280. https://doi.org/10.1080/02640414.2016.1265661

Theodorakis, Y., Hatzigeorgiadis, A., \& Chroni, S. (2008). Self-Talk: It Works, but How? Development and Preliminary Validation of the Functions of Self-Talk Questionnaire. Measurement in Physical Education and Exercise Science, 12(1), 10-30. https://doi.org/10.1080/10913670701715158

Thomas, O., Lane, A., \& Kingston, K. (2011). Defining and Contextualizing Robust Sport-Confidence. Journal of Applied Sport Psychology, 23(2), 189-208. https://doi.org/10.1080/10413200.2011.559519

Toma, M. (2017). Missed Shots at the Free-Throw Line: Analyzing the Determinants of Choking Under Pressure. Journal of Sports Economics, 18(6), 539-559. https://doi.org/10.1177/1527002515593779

Williams, A. M., \& Wigmore, T. (2020). The Best: How Elite Athletes Are Made. Nicholas Brealey Publishing.

Wilson, M. (2008). From processing efficiency to attentional control: A mechanistic account of the anxiety-performance relationship. International Review of Sport and Exercise Psychology, l(2), 184-201. https://doi.org/10.1080/17509840802400787 